\title{
A Time-based Passive Source Localization System for Narrow-band Signal
}

\author{
Zan Li, Torsten Braun, Desislava C. Dimitrova \\ Institute of Computer Science and Applied Mathematics, University of Bern, Bern - Switzerland \\ Email:1i@iam.unibe.ch, braun@iam.unibe.ch, dimitrova@iam.unibe.ch
}

\begin{abstract}
Time-based indoor localization has been investigated for several years but the accuracy of existing solutions is limited by several factors, e.g., imperfect synchronization, signal bandwidth and indoor environment. In this paper, we compare two time-based localization algorithms for narrow-band signals, i.e., multilateration and fingerprinting. First, we develop a new Linear Least Square (LLS) algorithm for Differential Time Difference Of Arrival (DTDOA). Second, fingerprinting is among the most successful approaches used for indoor localization and typically relies on the collection of measurements on signal strength over the area of interest. We propose an alternative by constructing fingerprints of fine-grained time information of the radio signal. We offer comprehensive analytical discussions on the feasibility of the approaches, which are backed up by evaluations in a software defined radio based IEEE 802.15.4 testbed. Our work contributes to research on localization with narrow-band signals. The results show that our proposed DTDOA-based LLS algorithm obviously improves the localization accuracy compared to traditional TDOA-based LLS algorithm but the accuracy is still limited because of the complex indoor environment. Furthermore, we show that time-based fingerprinting is a promising alternative to power-based fingerprinting.
\end{abstract}

\section{INTRODUCTION}

In the last years, research on the topic of indoor localization has become increasingly important, motivated by the shortcomings of the Global Positioning System (GPS) indoors but also by the attractiveness of business cases in various application fields such as Ambient Assisted Living (AAL), home automation, and security.

Wireless technologies have emerged as candidates for indoor localization due to their ubiquitousness. Particularly, IEEE 802.11 is currently the dominant local wireless network standard for short-range communication in indoor environments and is the leading technology for indoor localization [1]. IEEE 802.15.4, e.g., Zigbee, is another wireless standard for short-range communication. It is widely used in home automation, wireless sensor network and industry applications. It has attracted interests of researchers as an alternative to $\mathrm{WiFi}$ localization [2]. IEEE 802.15.4 utilizes narrow bandwidth, e.g., $2 \mathrm{MHz}$ bandwidth at $2.4 \mathrm{GHz}$ frequency band. This narrow-band property poses different challenges compared to IEEE 802.11 techniques, e.g., IEEE $802.11 \mathrm{n}$ with $20 \mathrm{MHz}$ and $40 \mathrm{MHz}$ bandwidth.

Indoor localization solutions can be divided into two categories, range-based and range-free. The most commonly used range-based localization technique is lateration, e.g., trilateration and multilateration. Range is defined as the distance between the target and Anchors Nodes (ANs). It can be obtained from various signal parameters such as time information or
Received Signal Strength (RSS). Lateration accuracy depends on the quality of input parameters and their vulnerability to propagation conditions. Hence, indoor environments with their complex multipath propagation patterns can significantly degrade the performance of the lateration technique. As an alternative to lateration for indoor spaces, fingerprinting was proposed for range-free localization and is a widely used technique. Traditionally, fingerprinting is relying on RSS. Although RSS can be easily obtained from off-the-shelf devices, it is a coarse Media Access Control (MAC) layer parameter. Therefore, some fine-grained physical layer parameters are recently investigated for fingerprinting to improve accuracy, e.g., Channel State Information (CSI) [1] and Channel Impulse Response (CIR) [3].

This paper contributes to the field of passive source localization, in which the localization algorithms are implemented at ANs. ANs just overhear the packets from the target to be localized. Passive source localization systems are attractive for third-party providers of positioning and monitoring services.

In our work, we select to work with Differential Time Difference Of Arrival (DTDOA). Compared to TDOA, DTDOA can eliminate the negative influence of imperfect synchronization between ANs by introducing a Reference Node (RN). In our previous work [4], we analyzed the ranging performance of DTDOA for narrow-band signals and proposed a geometric method to locate the target in an outdoor environment. In this paper, we propose two novel localization algorithms based on DTDOA for narrow-band signals. Our first contribution adds to range-based algorithms and employs a Linear Least Square (LLS) algorithm for DTDOA by introducing a new intermediate parameter. Our second contribution adds to rangefree localization and it is the first to propose and demonstrate the feasibility of DTDOA-based fingerprinting in indoor environments.

The two algorithmic contributions are supported by theoretical analysis and accuracy measurements in a real-world IEEE 802.15.4 testbed. The high reliability of our findings is backedup by a method to minimize the influence of outliers. The testbed is designed and implemented using software defined radio to overhear IEEE 802.15.4 signals and extract physical layer fine-grained information used for localization. Through a set of comprehensive measurements, we find that DTDOAbased LLS significantly improves the accuracy compared to TDOA-based LLS. However, the accuracy is still limited in indoor environments because of the severe multipath influence on the timestamps for narrow-band signals. TDOA-based fingerprinting achieves much better accuracy than LLS and is suitable to locate the target in indoor environments. Further- 
more, we compare the performances of DTDOA-based and RSS-based fingerprinting in different scenarios. The measurement results show that DTDOA-based fingerprinting achieves quite similar performance as power-based fingerprinting and in Non-Line Of Sight (NLOS) conditions DTDOA-based fingerprinting performs better than RSS.

In the remainder of the paper, we first describe some related work on time-based localization for narrow-band signals and traditional fingerprinting techniques in Section II. In Section III we theoretically analyze the difference of TDOA and DTDOA, introduce the proposed DTDOA-based LLS and fingerprinting algorithms, and provide a kernel based algorithm to mitigate the influence of outliers. Section IV presents our IEEE 802.15.4 testbed, in which packets from packet emitters are captured based on software defined radio techniques. In Section V, evaluations of DTDOA-based LLS and fingerprinting in two scenarios are presented. Finally, Section VI concludes the paper.

\section{RELATED WORK}

Indoor localization algorithms can be classified into two categories, range-based and range-free. Time-based localization is generally investigated for range-based localization, because of its high potential for accurate ranging. For rangefree localization, RSS-based fingerprinting has been widely investigated for decades and provides satisfying indoor accuracy.

\section{A. Time-based Multilateration for Narrow-band Signals}

Range-based localization comprises two phases including ranging and positioning. The accuracy of time-based ranging is influenced by time synchronization among the ANs and timestamp accuracy. Due to the high propagation speed of radio signals, time-based ranging requires strict time synchronization among ANs to achieve high accuracy. In [5], we found that the GPS synchronization offset is variable over time depending on the GPS signal reception conditions. This variance is impossible to predict and can cause positioning inaccuracy of around $60 \mathrm{~m}$. To eliminate the synchronization offset, we proposed to combine GPS synchronization and DTDOA, which introduces a reference node to compensate the GPS synchronization offset [4].

Timestamp accuracy is highly dependent on signal bandwidth. For example, due to its wide bandwidth, Ultra Wide Band (UWB) signals can achieve nanosecond accuracy for sample-based timestamps [6]. However, long symbol durations of narrow-band signals lead to low accurate samplebased timestamps. We proposed a physical layer timestamp based on symbol timing recovery for narrow-band signals, which can achieve nanosecond resolution [4]. Complex indoor propagation is another factor limiting ranging accuracy. The error sources include multipath propagation and shadowing. In narrow-band systems, signals from different propagation paths overlap in one symbol duration, making the detection of the direct propagation path nontrivial. Furthermore, shadowing could attenuate or completely block the direct propagation path, adding another bias to the range estimate. We demonstrated in [4] that indoor multipath propagation introduces a large biased value to the estimated timestamp.
At the positioning phase, multilateration is commonly used. In general, there are two different kinds of approaches to determine target locations based on multilateration, i.e., the geometric approach and the statistical approach. In [4], we introduced a geometric algorithm for DTDOA, which localizes the target at the intersection of hyperbolic curves. The algorithm is only feasible for small ranging errors. However, in indoor environments, the algorithm fails to find the intersection of the hyperbolic curves due to large ranging errors. As a statistical approach, Nonlinear Least Square (NLS) and LLS are two methods to solve nonlinear equations. LLS reorganizes nonlinear equations into a set of linear equations by introducing an intermediate variable so that real-time implementation is enabled [7].

\section{B. Fingerprinting}

Due to severe multipath effects, fingerprinting based on RSS is widely adopted in indoor environments. RADAR [8] presented an early fingerprinting system, which is comprised of offline and online phases. In the offline phase, RSS at multiple ANs are measured to build a RSS map. In the online phase, the K-Nearest Neighbours (KNN) algorithm is adopted to match the position of a target device to the RSS map. Horus [9] proposed to adopt a probabilistic algorithm in the online phase to improve accuracy.

Recently, some fine-grained factors at the physical layer other than RSS have been investigated to improve the accuracy of fingerprinting. In [3], CIR was analyzed with $200 \mathrm{MHz}$ bandwidth and neural networking training was adopted to improve accuracy. FIFS [1] was designed to use CSI for fingerprinting with WiFi signals. With these fine-grained channel information, the performance has been demonstrated to be impressively improved compared to RSS. However, CSI approaches for fingerprinting only work for Orthogonal Frequency Division Multiplexing (OFDM) systems, which benefit from frequency diversity. However, single carrier systems cannot be supported, e.g., Zigbee with Direct Sequence Spread Spectrum (DSSS) technique.

\section{DTDOA-BASED ALGORITHM DESIGN}

In this section, we first compare the theoretical performance of TDOA and DTDOA. Then, we introduce two proposed algorithms, i.e., DTDOA-based LLS and fingerprinting. Finally, we present a kernel based data aggregation method to mitigate the influence of outliers.

\section{A. TDOA Model}

TDOA is defined as the difference of arriving times between ANs for the same packet from a target. All ANs are synchronized by GPS signals. Given the factors influencing the accuracy of TDOA, we propose to decompose TDOA into four components [4].

$$
T_{\mathrm{TDOA}}=T_{\mathrm{d}}+T_{\mathrm{sh}}+T_{\mathrm{sy}}+n,
$$

where $T_{\text {TDOA }}$ is the overall TDOA value, $T_{\mathrm{d}}$ is the TDOA component related to the geometric distance, $T_{\mathrm{sh}}$ is the shadowing and multipath component, $T_{\text {sy }}$ is the synchronization offset component and $n$ is Gaussian noise. 


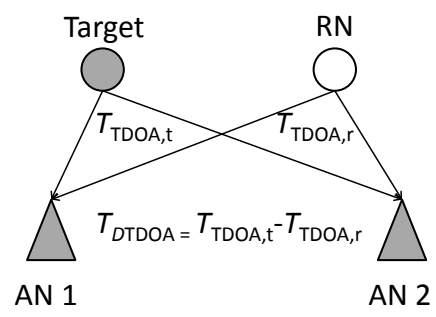

Fig. 1: DTDOA Operation

The components, $T_{\mathrm{d}}$ and $T_{\mathrm{sh}}$, are determined by the position of the target, and remain constant in a static environment. However, with GPS synchronization, the component $T_{\text {sy }}$ is unpredictably variable over time [4]. Therefore, the overall $T_{\text {TDOA }}$ in Equation (1) would be unpredictably variable as well, even if the target stays at the same position. Hence, $T_{\mathrm{TDOA}}$ is not only determined by the position of the target but also influenced by synchronization. $T_{\mathrm{TDOA}}$ is not a good measure for ranging and fingerprinting.

\section{B. DTDOA Model with GPS Synchronization}

DTDOA is defined as the difference of TDOA values for the target and an RN between the same pair of ANs. Figure 1 illustrates the calculation of DTDOA. Compared to a TDOA system, a RN is added to compensate for imperfect synchronization. The RN transmits a control packet immediately after overhearing the target's transmission [4].

Based on the TDOA model in Equation (1), the $T_{\text {TDOA }}$ values for the target and RN can be respectively defined as

$$
\begin{aligned}
& T_{\mathrm{TDOA}, \mathrm{t}}=T_{\mathrm{d}, \mathrm{t}}+T_{\mathrm{sh}, \mathrm{t}}+T_{\mathrm{sy}, \mathrm{t}}+n, \\
& T_{\mathrm{TDOA}, \mathrm{r}}=T_{\mathrm{d}, \mathrm{r}}+T_{\mathrm{sh}, \mathrm{r}}+T_{\mathrm{sy}, \mathrm{r}}+n,
\end{aligned}
$$

where $T_{\mathrm{d}, \mathrm{t}}, T_{\mathrm{sh}, \mathrm{t}}$ and $T_{\mathrm{sy}, \mathrm{t}}$ are the components for the target, and $T_{\mathrm{d}, \mathrm{r}}, T_{\mathrm{sh}, \mathrm{r}}$ and $T_{\mathrm{sy}, \mathrm{r}}$ are for the RN.

DTDOA $\left(T_{\mathrm{DTDOA}}\right)$ between the target and $\mathrm{RN}$ can be calculated as

$$
\begin{aligned}
T_{\mathrm{DTDOA}} & =T_{\mathrm{TDOA}, \mathrm{t}}-T_{\mathrm{TDOA}, \mathrm{r}} \\
& =\left(T_{\mathrm{d}, \mathrm{t}}-T_{\mathrm{d}, \mathrm{r}}\right)+\left(T_{\mathrm{sh}, \mathrm{t}}-T_{\mathrm{sh}, \mathrm{r}}\right)+\left(T_{\mathrm{sy}, \mathrm{t}}-T_{\mathrm{sy}, \mathrm{r}}\right)+n .
\end{aligned}
$$

The synchronization components $\left(T_{\mathrm{sh}, \mathrm{t}}\right.$ and $\left.T_{\mathrm{sh}, \mathrm{t}}\right)$ in Equation (4) can be eliminated via DTDOA in case that the variance of GPS synchronization is limited to sub-nanoseconds within a small time interval, e.g., 1 second [4]. Therefore, $T_{\text {DTDOA }}$ can be rewritten as

$$
T_{\mathrm{DTDOA}}=\left(T_{\mathrm{d}, \mathrm{t}}-T_{\mathrm{d}, \mathrm{r}}\right)+\left(T_{\mathrm{sh}, \mathrm{t}}-T_{\mathrm{sh}, \mathrm{r}}\right)+n .
$$

In Equation (5), besides the Gaussian noise, only components that are determined by the localization of the target are present. Therefore, the distribution of the $T_{\mathrm{DTDOA}}$ is expected to be Gaussian with mean value of $\left(T_{\mathrm{d}, \mathrm{t}}-T_{\mathrm{d}, \mathrm{r}}\right)+\left(T_{\mathrm{sh}, \mathrm{t}}-T_{\mathrm{sh}, \mathrm{r}}\right)$, which is only determined by the position of the target and RN. In consequence, $T_{\mathrm{DTDOA}}$ is a better candidate for ranging and fingerprinting.

\section{DTDOA-based Linear Least Square}

To design a range-based algorithm, the measured $T_{\mathrm{DTDOA}}$ values should be first converted into distance as $r_{\mathrm{DTDOA}, \mathrm{k}}=$ $T_{\text {DTDOA,k }} \cdot c$, where $c$ is the speed of light and $T_{\text {DTDOA,k }}$ is the measured $T_{\text {DTDOA }}$ between the $k$ th and the first ANs. After ranging, the estimated position $(\hat{x}, \hat{y})$ of the target can be found by the LLS algorithm, given the position $\left(x_{k}, y_{k}\right)$ of the $k$ th AN and position $\left(x_{R}, y_{R}\right)$ of the RN.

Without considering noise, we can obtain that

$r_{\mathrm{DTDOA}, \mathrm{k}}=$

$\left[\sqrt{\left(x-x_{k}\right)^{2}+\left(y-y_{k}\right)^{2}}-\sqrt{\left(x-x_{1}\right)^{2}+\left(y-y_{1}\right)^{2}}\right]-$

$\left[\sqrt{\left(x_{R}-x_{k}\right)^{2}+\left(y_{R}-y_{k}\right)^{2}}-\sqrt{\left(x_{R}-x_{1}\right)^{2}+\left(y_{R}-y_{1}\right)^{2}}\right]$,

where $(x, y)$ is the real position of the target. Two intermediate parameters, $R_{k}$ and $d_{1}$ are introduced. $R_{k}$ is the defined as the difference between the distances from the $\mathrm{RN}$ node to the $k$ th $\mathrm{AN}$ and the first AN:

$R_{k}=\sqrt{\left(x_{R}-x_{k}\right)^{2}+\left(y_{R}-y_{k}\right)^{2}}-\sqrt{\left(x_{R}-x_{1}\right)^{2}+\left(y_{R}-y_{1}\right)^{2}}$.

$d_{1}$ is defined as the distance from the location of the target to the first $\mathrm{AN}$ as $d_{1}=\sqrt{\left(x-x_{1}\right)^{2}+\left(y-y_{1}\right)^{2}}$.

Equation (6) can be rewritten into the following linear equation,

$$
\begin{aligned}
& \left(x-x_{1}\right)\left(x_{k}-x_{1}\right)+\left(y-y_{1}\right)\left(y_{k}-y_{1}\right)+\left(r_{\text {DTDOA }, \mathrm{k}}+R_{k}\right) d_{1} \\
& =\frac{1}{2}\left[\left(x_{k}-x_{1}\right)^{2}+\left(y_{k}-y_{1}\right)^{2}-\left(r_{\text {DTDOA }, \mathrm{k}}+R_{k}\right)^{2}\right] .
\end{aligned}
$$

Writing Equation (7) in matrix form gives,

$$
\begin{gathered}
\mathbf{G} \theta=\mathbf{h}, \\
\mathbf{G}=\left(\begin{array}{ccc}
x_{2}-x_{1} & y_{2}-y_{1} & r_{T D O A, 2}+R_{2} \\
\vdots & \vdots & \vdots \\
x_{M}-x_{1} & y_{M}-y_{1} & r_{T D O A, M}+R_{M}
\end{array}\right) \\
\mathbf{h}=\frac{1}{2}\left(\begin{array}{c}
\left(x_{2}-x_{1}\right)^{2}+\left(y_{2}-y_{1}\right)^{2}-\left(r_{T D O A, 2}+R_{2}\right)^{2} \\
\vdots \\
\left(x_{M}-x_{1}\right)^{2}+\left(y_{M}-y_{1}\right)^{2}-\left(r_{T D O A, M}+R_{M}\right)^{2}
\end{array}\right),
\end{gathered}
$$

where the parameter vector is $\theta=\left[x-x_{1}, y-y_{1}, d_{1}\right]^{T}$ and $M$ is the number of ANs. The location estimate is found as

$$
\begin{aligned}
\hat{\theta} & =\underset{\theta}{\operatorname{argmin}}\left[(\mathbf{G} \theta-\mathbf{h})^{T}(\mathbf{G} \theta-\mathbf{h})\right] \\
& =\left(\mathbf{G}^{T} \mathbf{G}\right)^{-1} \mathbf{G}^{T} \mathbf{h},
\end{aligned}
$$

and $\hat{\theta}=\left[\hat{x}-x_{1}, \hat{y}-y_{1}, d_{1}\right]^{T}$.

\section{DTDOA-based Fingerprinting}

The proposed fingerprinting method relies on two phases including an offline phase and an online phase. In the offline phase, a mobile signal emitter moves through the area of interest in different training positions and ANs record the radio parameters at each training position to form a radio map (database). In our work, DTDOA vectors (DTDOA $\mathbf{A}_{\mathbf{i}}$ ) are stored in the radio map. DTDOA $\mathbf{A}_{\mathbf{i}}$ comprises $T_{\mathrm{DTDOA}}$ values between different ANs at the $i$ th training position. 
Once the offline training phase is complete, the position of the target can be estimated by performing a radio scan and feeding the measured DTDOA vector to a localization algorithm. The easiest fingerprinting algorithm is the Weighted KNN (WKNN), which selects the $\mathrm{K}$ most nearest neighbours based on the Euclidean distance, and then returns the weighted average of these $\mathrm{K}$ locations as an estimate of the current location of the target. The Euclidean distances between DTDOA at the current position and all the training positions are firstly calculated as follows,

$$
e_{i}=\left\|\mathbf{D T D O A}-\mathbf{D T D O A}_{\mathbf{i}}\right\| \text {. }
$$

$\|\cdot\|$ indicates the norm value of a vector. Second, we set $K=3$, which means that the three positions in the radio map with the minimum $e_{i}$ are selected. Finally, the estimated position $(\hat{x}, \hat{y})$ of the target is calculated as

$$
(\hat{x}, \hat{y})=\sum_{i=1}^{3} \frac{w_{i}}{\sum_{j=1}^{3} w_{j}}\left(x_{i}, y_{i}\right),
$$

where $w_{i}=\frac{1}{e_{i}}$, and $\left(x_{i}, y_{i}\right)$ are the coordinates of the $i$ th training position.

\section{E. KDE-based Data Aggregation}

Outliers that are caused by measurement errors can obviously deteriorate the performance of localization algorithms. We propose to apply Kernel Density Estimation (KDE) to aggregate the $T_{\mathrm{DTDOA}}$ values from different packets to mitigate the influence of outliers. The benefit of KDE is that it can estimate the density directly from the data without assuming a particular form for the underlying distribution.

Assuming that we have $n$ independent observations of $T_{\text {DTDOA }}, \mathbf{X}=\left[x_{1}, x_{2}, \ldots, x_{n}\right]$, the kernel density estimator $\hat{f}_{h}(x)$ of the density value $f(x)$ at point $x$ is defined as

$$
\widehat{f}_{h}(x)=\frac{1}{n h} \sum_{i=1}^{n} K\left(\frac{x_{i}-x}{h}\right),
$$

where $K(u)$ denotes the kernel function, and $h$ denotes the bandwidth. In our work, we adopt a Gaussian kernel, $K(u)=$ $\frac{1}{\sqrt{2 \pi}} \exp \left(-\frac{1}{2} u^{2}\right)$.

Because of its Gaussian distribution with symmetric shape, DTDOA can be estimated by finding the DTDOA value with maximum density in the KDE. Therefore, we propose to first adopt the KDE method to calculate $\widehat{f}_{h}(x)$ (as in Equation (12)). The DTDOA is then estimated as,

$$
\hat{T}_{\mathrm{DTDOA}}=\underset{T_{\mathrm{DTDOA}}}{\operatorname{argmax}} \widehat{f}_{h}\left(T_{\mathrm{DTDOA}}\right) \text {. }
$$

$\hat{T}_{\mathrm{DTDOA}}$ is the aggregated DTDOA value.

\section{LocAlization TESTBED DESIGN FOR IEEE 802.15.4 SIGNALS}

To evaluate our proposed system, we design a passive system based on Software Defined Radio (SDR), which can overhear IEEE 802.15.4 signals, and accurately timestamp the captured messages. As signal capturing hardware, the Universal Software Radio Peripheral (USRP) with model N210 is used. Signal processing is implemented in GNU Radio [10],

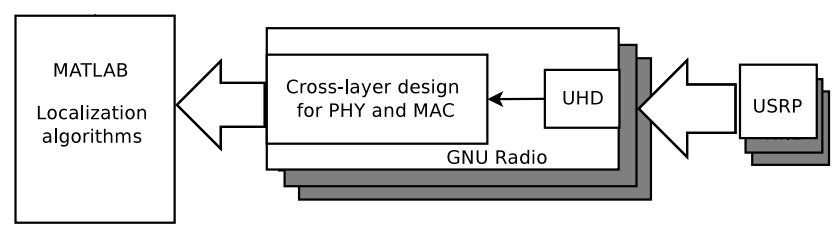

(a) Overall Testbed Structure

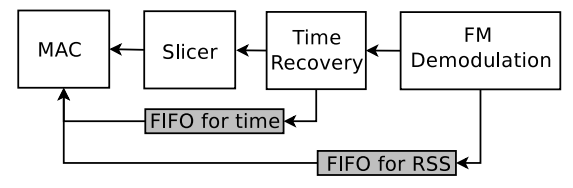

(b) Cross-layer Design for PHY and MAC Block

Fig. 2: Testbed Structure

which is utilized for demodulation and packet reconstruction. All USRPs in our work are synchronized by GPS receivers.

As shown in Figure 2(a), the testbed comprises three main components, i.e., USRP for signal capturing, GNU Radio for signal processing, and MATLAB for the localization algorithms. The physical layer parameters for localization, e.g., RSS and timestamp, are extracted in GNU Radio and passed to MATLAB processing. We adopt an advanced method to achieve sub-sample timestamps, which can estimate the subsample time delay between two output samples from one USRP by using symbol timing recovery from the signal processing chain [4]. KDE-based data aggregation, DTDOAbased LLS and fingerprinting are implemented in MATLAB as localization algorithms.

The authors of [11] provided an IEEE 802.15.4 decoding system, in which physical and MAC layers are separated into different blocks. To obtain the physical layer information from each packet, an approach called stream tags is commonly adopted to attach these information to each sample and pass to the MAC layer block. This method significantly increases the processing load and results in overflows with sampling rate of $4 \mathrm{MHz}$ in a desktop with i5 $\mathrm{CPU}(3.3 \mathrm{GHz})$. Because of overflows, many samples are lost and hence the packet decoding rate is dramatically reduced.

In our testbed, we implement a cross-layer structure integrating physical and MAC layers into one GNU Radio block, which can more efficiently pass the parameters from physical layer to MAC layer and solve the overflow problem. At the physical layer, we implement similar methods as in [11] Figure 2(b) indicates the structure, where an MSK (MinimumShift Keying) demodulation method is implemented to decode IEEE 802.15.4 signals. The power for each sample is obtained before FM (Frequency Modulation) demodulation and subsample timestamps are obtained in the time recovery part. The power and sub-sample timestamp for each sample are stored in two FIFO (First Input First Output) buffers. As soon as the packet is reconstructed at the MAC layer, the power and subsample timestamp are obtained by averaging all the samples in a packet.

Based on the proposed cross-layer design, we are able to reduce CPU usage by more than half and avoid overflows. We tested our decoding methods with a TelosB node as a 


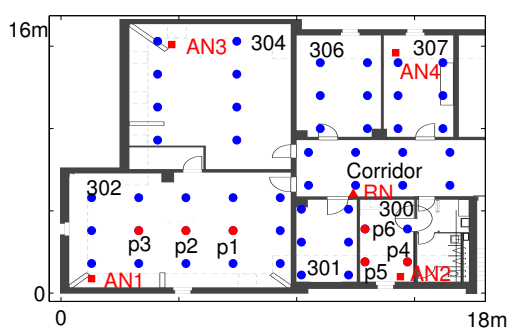

(a) Radio Map on the Third Floor and Testing Positions for LLS

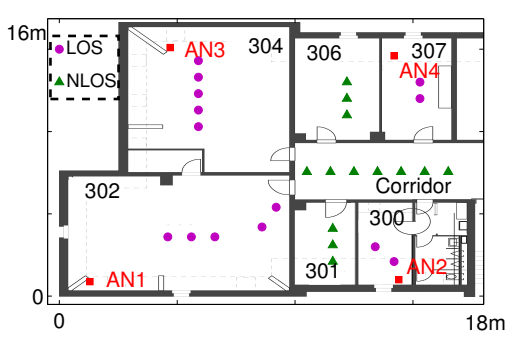

(b) Testing Positions on the Third Floor

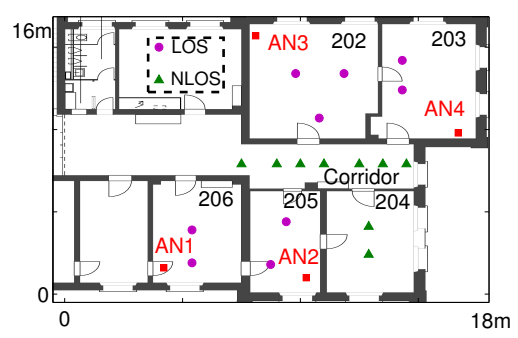

(c) Testing Positions on the Second Floor

Fig. 3: Measurement Setup

signal emitter, which includes a CC2420 radio transceiver. We configured the CC2420 radio transceiver to the maximum transmission power level (level 31). To test the performance of the testbed, the distance between the USRP receiver and TelosB emitter with LOS connection was changed from $0.5 \mathrm{~m}$ to $11.5 \mathrm{~m}$ at $1 \mathrm{~m}$ steps. For each distance 600 packets of the target were analyzed and we were able to decode more than $99.4 \%$ of the transmitted packets.

\section{Localization Measurements}

Based on the proposed IEEE 802.15.4 testbed, we conducted comprehensive measurements to evaluate the proposed DTDOA-based LLS and fingerprinting methods.

\section{A. Measurement Setup}

Evaluation measurements were conducted at two test locations, i.e., the second and third floor of the IAM building at University of Bern. The two locations have different layouts as shown in Figure 3(c) and Figure 3(b). The second floor is our working area and people are moving during working hours. The third floor is mainly occupied by lab and less frequently used seminar rooms. Hence, the environment on the third floor is more stable.

Four ANs were deployed to capture IEEE 802.15.4 signals from TelosB nodes. A RN (TelosB node) was placed in the system and used to calculate $T_{\mathrm{DTDOA}}$ values. The target TelosB node periodically broadcasts packets. As soon as the RN has received one packet from the target, it retransmits the packet. The ANs overhear packets from both the target and $\mathrm{RN}$ to calculate the corresponding $T_{\mathrm{DTDOA}}$. The target was configured to transmit five packets per second. The data collection time at each test position was one minute.

First, as a proof of concept, several preliminary measurements were conducted on the third floor of the IAM building to analyze DTDOA and TDOA regarding the following three aspects: distribution, variance, and Euclidean distance among neighbours.

Second, 53 positions (Figure 3(a)) on the third floor were tested to analyze the performance of DTDOA-based ranging and LLS. Moreover, we compare the performance of DTDOAbased LLS to TDOA-based LLS.

Third, the proposed DTDOA-based fingerprinting was tested on both the second and third floor. Before testing, two radio maps were created in these two locations during the weekend when there was no change of the layouts and no people movement. Figure 3(a) shows an example of the radio map on the third floor, in which training positions are separated by approximately $2 m$.

For online tests of the fingerprinting algorithms, the first measurements in both scenarios were taken one day after the creation of radio map at the same weekend. The second measurements were conducted five days later during working hours. 18 test positions were randomly selected on the second floor as shown in Figure 3(c). Among them, 9 positions were located in areas, where the target has LOS connection to one of the ANs, and are thus referred to LOS areas. 9 positions were in areas, where the target has no LOS connection to any $\mathrm{AN}$, and are referred to NLOS areas. 27 positions on the third floor were tested as shown in Figure 3(b). Among them, 14 positions were in the LOS areas and 13 positions were in the NLOS areas.

\section{B. Preliminary DTDOA Analysis based on Measurements}

Distribution. We analyze the distribution of $T_{\mathrm{TDOA}}$ and $T_{\text {DTDOA }}$ values for a single position. Figure 4(a) and 4(b) show the normalized histogram and estimated Probability Density Function (PDF) of $T_{\mathrm{TDOA}}$ and $T_{\mathrm{DTDOA}}$ respectively. According to the estimated PDF, the distribution of $T_{\text {DTDOA }}$ fits better to a Gaussian distribution than $T_{\mathrm{TDOA}}$, whose PDF is influenced by imperfect synchronization. This finding supports our claim in Section III-B.

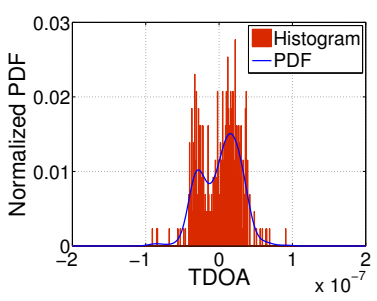

(a) Distribution of TDOA

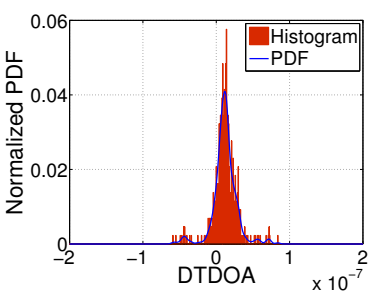

(b) Distribution of DTDOA
Fig. 4: Distribution of TDOA and DTDOA

Variance. Figure 5 shows a DTDOA measurement between two ANs for thirty minutes with the target moving from one position to another and then back but remaining static for 
ten minutes at each position. As shown in the figure, the mean DTDOA value does not change significantly for a single position but visibly differs from one position to the next. This finding further supports our analysis from Section III-B.

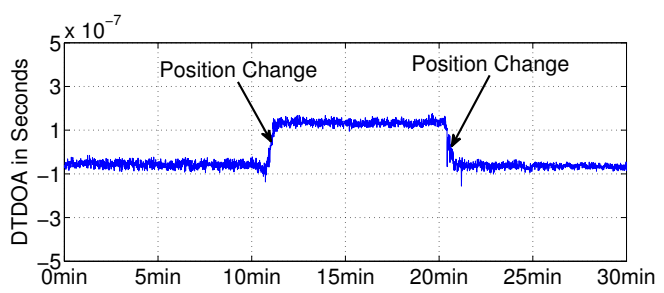

Fig. 5: DTDOA Change at Different Positions

Euclidean Distance among Neighbours. In order to apply fingerprinting $(\mathrm{WKNN})$, the DTDOA vectors $\left(\mathbf{D T D O A}_{\mathbf{i}}\right.$ in Section III-D) among the neighbours should have lower Euclidean distance than the positions in far away areas. Therefore, we analyze DTDOA vectors at six different positions (P1 to P6 in Figure 3(a)). Figure 6 shows the DTDOA vectors at the six positions, where $y$-axis is the $T_{\mathrm{DTDOA}}$ values and $\mathrm{x}$-axis indicates the ANs from which the $T_{\text {DTDOA }}$ is obtained. For example, $\mathrm{AN}(1,2)$ means that the $T_{\mathrm{DTDOA}}$ value is calculated between the first $\mathrm{AN}$ and the second AN. Each single vector comprises six $T_{\text {DTDOA }}$ values between different ANs. As shown in Figure 6, the DTDOA vectors in nearby areas have smaller gaps among each other, which means lower Euclidean distance.

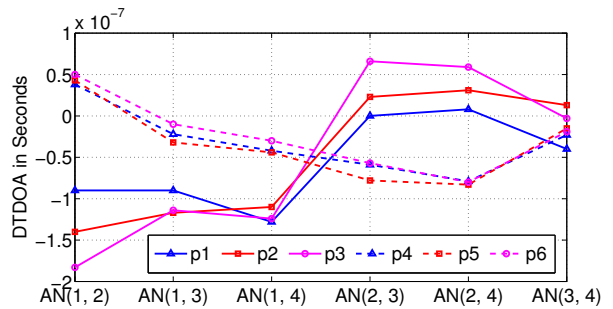

Fig. 6: DTDOA Vectors at Different Positions

Based on the preliminary evaluations, $T_{\mathrm{DTDOA}}$ is only determined by the target position but not influenced by synchronization. Furthermore, DTDOA vectors in nearby areas have lower Euclidean distance between each other. Both findings support the feasibility of DTDOA fingerprinting.

\section{Measurement Results for DTDOA-based LLS}

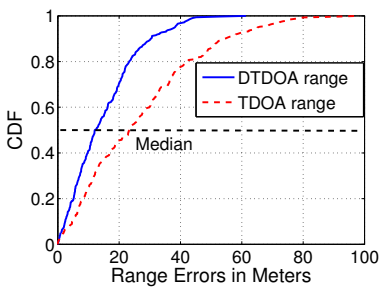

(a) CDF of Ranging Errors

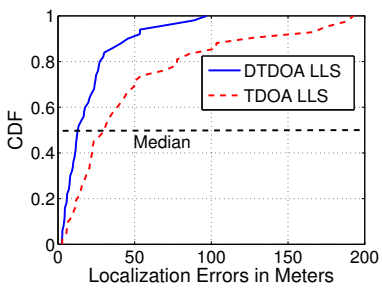

(b) CDF of Localization Errors
Fig. 7: Ranging and Localization Errors
Figure 7(a) shows the Cumulative Distributed Function (CDF) of ranging errors based on the DTDOA and TDOA methods. As shown in Figure 7(a), DTDOA improves the median ranging error by $50 \%$ (from $22 \mathrm{~m}$ to $11 \mathrm{~m}$ ) compared to TDOA-based ranging. Figure 7(b) indicates CDF of localization errors for DTDOA and TDOA-based LLS. As shown in Figure 7(b), the improvement of median error by DTDOAbased LLS is around $57 \%$ (from $30 \mathrm{~m}$ to $13 \mathrm{~m}$ ) compared to TDOA-based LLS. In addition, the overall improvement of maximum ranging error and localization error is over $50 \%$.

We show that DTDOA achieves higher accuracy than TDOA by eliminating the influence of imperfect synchronization. However, multipath and NLOS propagation still introduce a large error to DTDOA-based ranging for narrow-band signals. DTDOA-based LLS is sensitive to ranging errors and hence it is still challenging to achieve accurate localization in a complex indoor environment.

\section{Measurement Results for DTDOA-based Fingerprinting}

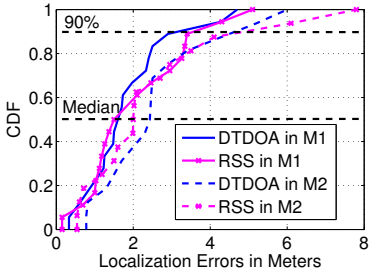

(a) CDF of Localization Errors

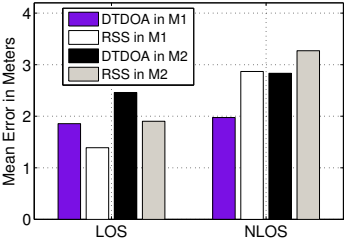

(b) Mean Errors in LOS and NLOS
Fig. 8: Localization Errors with WKNN on the Second Floor

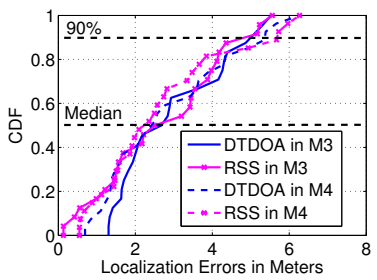

(a) $\mathrm{CDF}$ of Localization Errors (b) Mean Errors in LOS and NLOS

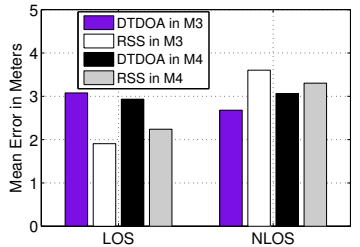

Fig. 9: Localization Errors with WKNN on the Third Floor

TABLE I: Measurement Notation

\begin{tabular}{|c|c|c|c|}
\hline Notation & Floor No. & Time & Environment \\
\hline M1 & Second & 1 day later & Static \\
\hline M2 & Second & 5 days later & Dynamic \\
\hline M3 & Third & 1 day later & Static \\
\hline M4 & Third & 5 days later & Static \\
\hline
\end{tabular}

TABLE II: Localization Errors Statistics

\begin{tabular}{|c|c|c|c|c|}
\cline { 2 - 5 } \multicolumn{1}{c|}{} & \multicolumn{2}{c|}{ RSS-based WKNN } & \multicolumn{2}{c|}{ DTDOA-based WKNN } \\
\hline Measurements & Mean & SD & Mean & SD \\
\hline M1 & $2.13 \mathrm{~m}$ & $1.35 \mathrm{~m}$ & $1.92 \mathrm{~m}$ & $1.15 \mathrm{~m}$ \\
\hline M2 & $2.59 \mathrm{~m}$ & $2 \mathrm{~m}$ & $2.65 \mathrm{~m}$ & $1.47 \mathrm{~m}$ \\
\hline M3 & $2.72 \mathrm{~m}$ & $1.72 \mathrm{~m}$ & $2.89 \mathrm{~m}$ & $1.65 \mathrm{~m}$ \\
\hline M4 & $2.8 \mathrm{~m}$ & $1.6 \mathrm{~m}$ & $3 \mathrm{~m}$ & $1.4 \mathrm{~m}$ \\
\hline
\end{tabular}

To achieve accurate indoor localization with time-based information, i.e., DTDOA, we further evaluate our proposed 
DTDOA-based fingerprinting algorithm and compare the performance to RSS-based fingerprinting. The measurement notations in Table I are used in the following section.

Accuracy. First, Table II summarizes the mean localization errors and Standard Deviations (SD) of the four measurements. For DTDOA-based fingerprinting, the mean errors in the four measurements are smaller than $3 \mathrm{~m}$. Compared to rangebased localization, i.e., DTDOA-based LLS, DTDOA-based fingerprinting achieves much better accuracy and is thus better suited for indoor localization. DTDOA-based and RSS-based fingerprinting algorithms achieve quite similar performance in terms of mean error in all four measurements. Figures 8(a) and 9(a) summarize CDFs of the localization errors. Similar as the observations based on mean error, CDFs indicate that DTDOA-based and RSS-based fingerprinting achieve similar performance in all four measurements.

Second, we further investigate and compare the performance of DTDOA-based and RSS-based fingerprinting in the LOS and NLOS areas. Figure 8(b) shows the mean errors for DTDOA-based and RSS-based fingerprinting in the LOS and NLOS areas on the second floor. Figure 9(b) is for the third floor. As shown in Figures 8(b) and 9(b), DTDOA-based and RSS-based fingerprinting algorithms achieve different performance in the LOS and NLOS areas. In the LOS areas, RSSbased fingerprinting achieves higher accuracy than DTDOAbased fingerprinting. The reason is that in the RSS vector there is a strong RSS component from the AN with LOS connection to the target, and therefore RSS-based fingerprinting can more accurately select the neighbours. However, in the NLOS areas, there is no strong RSS component in the RSS vector and DTDOA-based fingerprinting generally achieves better performance in all four measurements. Take measurement M1 as an example. In the LOS areas, the RSS-based fingerprinting with mean error of $1.39 \mathrm{~m}$ outperforms DTDOA-based fingerprinting with mean error of $1.86 \mathrm{~m}$. In the NLOS areas, DTDOAbased fingerprinting achieves mean error of $1.98 \mathrm{~m}$, which is $0.89 \mathrm{~m}$ better than RSS-based fingerprinting.

Stability. In addition, we analyze the time stability of fingerprinting algorithms, i.e., the performance in the same scenario but at different measurement time. For the measurements (M1 and M2) on the second floor, the environment is dynamic during working hours. Measurement M1 was conducted one day after creating the radio map but M2 was five days later. As shown in Figure 8(a), both DTDOA-based and RSS-based fingerprinting in measurement M2 achieve $90 \%$ localization error below $4.6 \mathrm{~m}$, which significantly deteriorates compared to the measurement M1 (3.2m for DTDOA-based fingerprinting and $3.6 \mathrm{~m}$ for RSS-based fingerprinting). The reason is that during working hours people move in offices and the layout of the surrounding environment also changes, e.g., doors become open or closed. These factors influence the accuracy for matching algorithms, i.e., WKNN, to find the correct neighbours based on the original fingerprinting database. Different from the measurements M1 and M2, the performance of DTDOAbased and RSS-based fingerprinting algorithms does not significantly deteriorate in the measurement M4 compared to M3 (Figure 9(a)), because the environment on the third floor was quite stable and the layout did not change.

\section{CONCLUSIONS}

A novel passive source localization system is presented in this paper, which utilizes fine-grained time information at the physical layer for multilateration and fingerprinting in a narrow-band system. Our system is able to passively capture IEEE 802.15.4 packets and obtain physical layer high resolution timestamps based on a software defined radio technique. DTDOA-based LLS and fingerprinting algorithms have been evaluated. Through experiments, we demonstrated that DTDOA-based LLS significantly improves localization accuracy, compared to TDOA-based LLS. However, in a complex indoor environment, NLOS propagation still introduces large errors. We further evaluated the DTDOA-based fingerprinting and demonstrated that in the narrow-band system DTDOAbased fingerprinting significantly outperforms LLS and is able to locate the target in an indoor environment with a mean error of $3 \mathrm{~m}$.

\section{ACKNOWLEDGEMENT}

This work was partly supported by the COST AAPELE Action IC1303.

\section{REFERENCES}

[1] K. Wu, J. Xiao, Y. Yi, D. Chen, X. Luo, and L. Ni, "Csi-based indoor localization," Parallel and Distributed Systems, IEEE Transactions on, vol. 24, no. 7, pp. 1300-1309, July 2013.

[2] D. Macii, A. Colombo, P. Pivato, and D. Fontanelli, "A data fusion technique for wireless ranging performance improvement," Instrumentation and Measurement, IEEE Transactions on, vol. 62, no. 1, pp. 27-37, Jan 2013.

[3] N. Khanbashi, N. Alsindi, S. Al-Araji, N. Ali, and J. Aweya, "Performance evaluation of cir based location fingerprinting," in Personal Indoor and Mobile Radio Communications (PIMRC), 2012 IEEE 23rd International Symposium on, Sept 2012, pp. 2466-2471.

[4] Z. Li, D. Dimitrova, D. Raluy, and T. Braun, "Tdoa for narrowband signal with low sampling rate and imperfect synchronization," in Wireless and Mobile Networking Conference (WMNC), 2014 7th IFIP, May 2014, pp. 1-8.

[5] Z. Li, D. Dimitrova, T. Braun, and D. Rosario, "Highly accurate evaluation of gps synchronization for tdoa localization," in Wireless Days (WD), 2013 IFIP, Nov 2013, pp. 1-3.

[6] D. Dardari, A. Conti, U. Ferner, A. Giorgetti, and M. Win, "Ranging with ultrawide bandwidth signals in multipath environments," Proceedings of the IEEE, vol. 97, no. 2, pp. 404-426, 2009.

[7] K. W. Cheung, H. C. So, W.-K. Ma, and Y. T. Chan, "A constrained least squares approach to mobile positioning: Algorithms and optimality," EURASIP J. Appl. Signal Process., vol. 2006, pp. 150-150, Jan. 2006.

[8] P. Bahl and V. Padmanabhan, "Radar: an in-building rf-based user location and tracking system," in INFOCOM 2000. Nineteenth Annual Joint Conference of the IEEE Computer and Communications Societies. Proceedings. IEEE, vol. 2, 2000, pp. 775-784 vol.2.

[9] M. Youssef and A. Agrawala, "The horus wlan location determination system," in Proceedings of the 3rd International Conference on Mobile Systems, Applications, and Services, ser. MobiSys '05, 2005, pp. 205218.

[10] Gnu radio website. [Online]. Available: http://gnuradio.org/redmine/wiki/gnuradio

[11] L. Choong, "Multi-channel ieee 802.15.4 packet capture using software defined radio," 2009. 